\title{
Sensory Representations Supporting Memory Specificity: Age Effects on Behavioral and Neural Discriminability
}

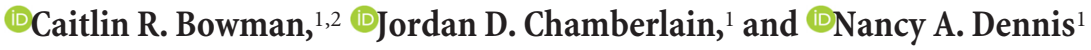 \\ ${ }^{1}$ Department of Psychology, The Pennsylvania State University, University Park, Pennsylvania 16802, and 2Department of Psychology, University of \\ Oregon, Eugene, Oregon 97403
}

Older adults' difficulty in distinguishing between old and new information contributes to memory decline, which may occur because older adults are less likely than young adults to retrieve specific sensory details necessary to distinguish between similar items. In male and female human subjects, the present study measured the extent of age differences in the specificity of memory representations using a false memory paradigm in which studied items were linked to retrieval items at multiple levels of similarity. Older adults showed poorer behavioral discrimination than young adults, driven primarily by false recognition of lures that differed from targets only in perceptual details. Patterns of activation across several regions within ventral visual cortex could be used to distinguish between targets and lures when they differed in both perceptual details and a semantic label. However, of ventral visual regions, only signals in the midline occipital cortex could be used to distinguish targets from lures when they differed only in perceptual details. Although there was an overall age deficit for this neural discrimination in this region, the positive relationship between neural and behavioral discriminability did not differ across age groups. In contrast, age moderated the relationship between neural and behavioral discriminability in lateral occipital and fusiform cortices, suggesting that activation patterns within these regions represent different types of information in each age group. Therefore, the quality of perceptual signals is a key contributor to memory discrimination across age groups, with evidence that age differences in the nature of representations emerges outside early visual cortex.

Key words: aging; false memory; functional MRI; long-term memory; multivariate pattern analysis

Significance Statement

Age-related memory decline is due in part to older adults' difficulties in discriminating between old and new information. We tested whether this deficit arises from lack of specificity in the sensory representations underlying older adults' recognition judgments. Using pattern classification analyses in ventral visual cortices, we found that signals in a region early in the visual stream could distinguish between targets and lures at the highest level of similarity. The discriminability of targets and lures in this region was positively related to behavioral discriminability across age groups despite an overall age deficit in classification accuracy. Together, results showed that older adults' memory deficits are related to reduced discriminability of cognitive processes (old/new recognition) in portions of visual cortex.

\section{Introduction}

Aging is often associated with increased forgetfulness, but increases in false memories are also a substantial contributor to

Received July 27, 2018; revised Jan. 9, 2019; accepted Jan. 10, 2019.

Author contributions: C.R.B. wrote the first draft of the paper; C.R.B., J.D.C., and N.A.D. edited the paper; C.R.B. and N.A.D. designed research; C.R.B. performed research; C.R.B. and J.D.C. analyzed data; C.R.B. wrote the paper.

This work was supported in part by the National Science Foundation (Grant BCS1025709 to N.A.D.) and the American Psychological Association and Penn State's Research and Graduate Studies Office (dissertation awards to C.R.B.) Support during manuscript preparation was also provided by the National Institutes of Health's National Institute on Aging (Grant F32 AG054204 to C.R.B.). We thank the Penn State Social, Life, and Engineering Science Imaging Center 3 T MRI facility.

The authors declare no competing financial interests.

Correspondence should be addressed to Nancy A. Dennis at nad12@psu.edu.

https://doi.org/10.1523/JNEUROSCI.2022-18.2019

Copyright $\odot 2019$ the authors $\quad 0270-6474 / 19 / 392265-11 \$ 15.00 / 0$ age-related memory decline (Koutstaal and Schacter, 1997; Balota et al., 1999; McCabe et al., 2009; McCormick-Huhn et al., 2018). Laboratory studies often elicit false memories by presenting new information at retrieval that shares features with items from study (Deese, 1959; Roediger et al., 1995). This overlap between new and old information means that memory for studied information must be highly specific to discriminate between the two types of information during retrieval. Therefore, age differences in the specificity of representations underlying memory decisions may be an important component of age-related increases in false memories.

Neuroimaging studies in young adults have shown that, whereas much of the brain does not show differences in univariate activation between true and false memories (Kim and Cabeza, 
2007; Dennis et al., 2012), portions of visual cortex are some of the few that sometimes show greater activation for true compared with false memories (Slotnick and Schacter, 2004; Dennis et al., 2012). Visual activation for true memories may reflect retrieval of specific perceptual details associated with items that were truly encountered in the past. Aging studies have shown that, compared with young adults, older adults exhibit reduced activation in early visual regions associated with true memories (Cabeza et al., 2004; Davis et al., 2008; Dennis et al., 2014). Such reductions in activation suggest that perceptual signals that help to make subtle memory discriminations in young adults may diminish with age (Koutstaal, 2003).

Dedifferentiation of visual representations may also contribute to age-related memory decline. During perception, regions of ventral visual cortex that respond preferentially to a given class of visual stimuli show less distinctive activation in older compared with young adults (Park et al., 2004, 2012). For example, in older adults, patterns of activity in ventral visual cortex corresponding to faces become less similar to one another and more similar to patterns associated with other stimulus classes, such as houses (Carp et al., 2011). Critically, memory retrieval, especially in false memory paradigms, relies on the ability to represent items with enough specificity to distinguish between old and new items that often come from a single stimulus class. However, the degree of age-related specificity decline remains an open question that can be addressed with a task in which retrieval items vary in their similarity to studied items. With such a design, we can assess how aging affects the discriminability of neural representations for old and new items across different levels of overlap between retrieval items.

To assess age effects on the specificity of neural representations, we used a false memory paradigm in which retrieval items corresponded to studied items across multiple levels of similarity: exact same item (target), new exemplar of a studied item (item lure), and new item from a studied category (thematic lure). We used classifier-based multivoxel pattern analysis (MVPA) to measure the discriminability of neural representations within ventral visual cortex in young and older adults. Specifically, we tested whether regions of ventral visual cortex distinguish between targets and lures across multiple levels of target-lure similarity and if the neural discriminability of targets and lures relates to memory discriminability in behavior. Because regions early in the ventral stream are hypothesized to support recapitulation of episodic details, we predicted that patterns of activation in midline occipital cortex (containing early visual regions) would be important for making subtle discriminations between similar items. Further, we predicted that older adults would show reduced discriminability of neural patterns for these subtle discriminations, consistent with the most prominent age deficit in behavior.

\section{Materials and Methods}

\section{Participants}

Twenty-seven young adults and 27 older adults were recruited from The Pennsylvania State University (Penn State) and the surrounding community and received monetary compensation for their participation. All participants were right-handed, native English speakers who were screened for contraindications for MRI, as well as neurological and psychiatric disorders. Two young adults were excluded from analyses due to failure to complete the task ( 1 subject) and movement in excess of 2.5 $\mathrm{mm}$ within a run ( 1 subject), leaving data from 25 young adults included in all analyses $(\mathrm{M}=22.32$ years old, $\mathrm{SD}=3.28$ years, range $=18-31 ; 17$ females). Other univariate analyses from the young adult group have been presented previously (Bowman and Dennis, 2016; Bowman et al.,
Table 1. Participant demographic information

\begin{tabular}{lcc}
\hline & Young $(n=25)$ & Older $(n=23)$ \\
\hline Age & $22.32(3.28)$ & $71.04(8.04)$ \\
Education (years) & $14.96(1.69)$ & $16.92(2.04)$ \\
Cognitive assessment tasks & \\
$\quad$ MMSE & $29.65(0.65)$ \\
WAIS-III & \\
$\quad$ Symbol search & $13.00(2.76)$ \\
$\quad$ Digit symbol coding & $12.83(3.01)$ \\
$\quad$ Symbol copy & $111.78(19.41)$ \\
$\quad$ Digit span & $13.39(3.16)$ \\
$\quad$ Arithmetic & $11.39(3.17)$ \\
$\quad$ Letter number sequencing & $12.69(4.18)$ \\
$\quad$ Vocabulary & $12.91(2.50)$ \\
BDI & $1(3.74)$ \\
GDS & $0.86(1.08)$ \\
\hline
\end{tabular}

Data are shown as means (SD) of demographic variables and cognitive assessment tasks separated by age group. MMSE, Mini-Mental State Exam; WAIS, Weschler Adult Intelligence Scale; BDI, Beck's Depression Inventory; GDS, Geriatric Depression Scale.

2017). Four older adults were excluded from analyses due to technical issues during data collection (2 subjects) and movement in excess of 2.5 $\mathrm{mm}$ within a run ( 2 subjects), leaving data from 23 older adults included in all analyses $(\mathrm{M}=71.04$ years old, $\mathrm{SD}=8.04$ years, range $=60-84 ; 12$ females). Before the study, older adults completed a series of neuropsychological tests, including the Mini Mental State Exam (Folstein et al., 1983), subtests from the Weschler Adult Intelligence Scale (Symbol Search, Digit Symbol Coding, Symbol Copy, Digit Span, Letter Number Sequence, Arithmetic, and Vocabulary; Wechsler, 1997), and either the Beck's Depression Inventory or the Geriatric Depression Scale (Sheikh and Yesavage, 1986). All participants performed within the normal range for their age group in each test (see Table 1 for individual scores). All participants completed written informed consent and all experimental procedures were approved by Penn State's Office of Research Protections.

\section{Stimuli}

Stimuli consisted of 316 images of common objects collected from the Band of Standardized Stimuli database (Brodeur et al., 2010) and from an internet image search. Images were cropped and resized to $\sim 400 \times 400$ pixels and equated for resolution. Images were displayed at a screen resolution of $1024(\mathrm{H}) \times 768(\mathrm{~V})$ at $75 \mathrm{~Hz}$. At the viewing distance of 143 $\mathrm{cm}$, the display area was $20^{\circ}(\mathrm{H})^{\circ} 16^{\circ}(\mathrm{V})$ with experimental stimuli subtending $5^{\circ}(\mathrm{H}) \times 4^{\circ}(\mathrm{V})$.

Encoding items included 96 images, each an exemplar from a distinct category or conceptual theme. At retrieval, four types of items were presented: targets, item lures, thematic lures, and novel lures (see Fig. 1 for examples). Item lures were an alternative exemplar of the same item presented at encoding. Thematic lures were a new item within the same general category or theme of an item presented at encoding. Novel lures were items drawn from categories not presented during encoding. A norming study was conducted to verify that item lures were perceived as more similar to targets than were thematic lures and that novel lures were perceived as unrelated to all targets. Using a 1-5 scale, 15 participants rated target-item lure and target-thematic lure pairs in terms of how similar the members of the pair were to one another, including similarities in physical appearance, use, category, or any other important characteristic. In addition, 30 participants saw individual novel lures and rated how similar each was to all targets, item lures, and thematic lures. Results showed higher similarity between item lures and targets $(\mathrm{M}=$ $4.71, \mathrm{SD}=0.22)$ than between targets and thematic lures $(\mathrm{M}=3.61$, $\left.\mathrm{SD}=0.38 ; t_{(14)}=14.40, p<0.001, d=3.72\right)$. Additionally, similarity ratings of novel lures compared with all targets, item, and thematic lures $(\mathrm{M}=1.79, \mathrm{SD}=0.49)$ were lower than both target-item lure pairs $\left(t_{(43)}=21.79, p<0.001, d=3.25\right)$ and target-thematic lure pairs $\left(t_{(43)}=12.60, p<0.001, d=1.82\right)$. 
Retrieval items related to encoding items

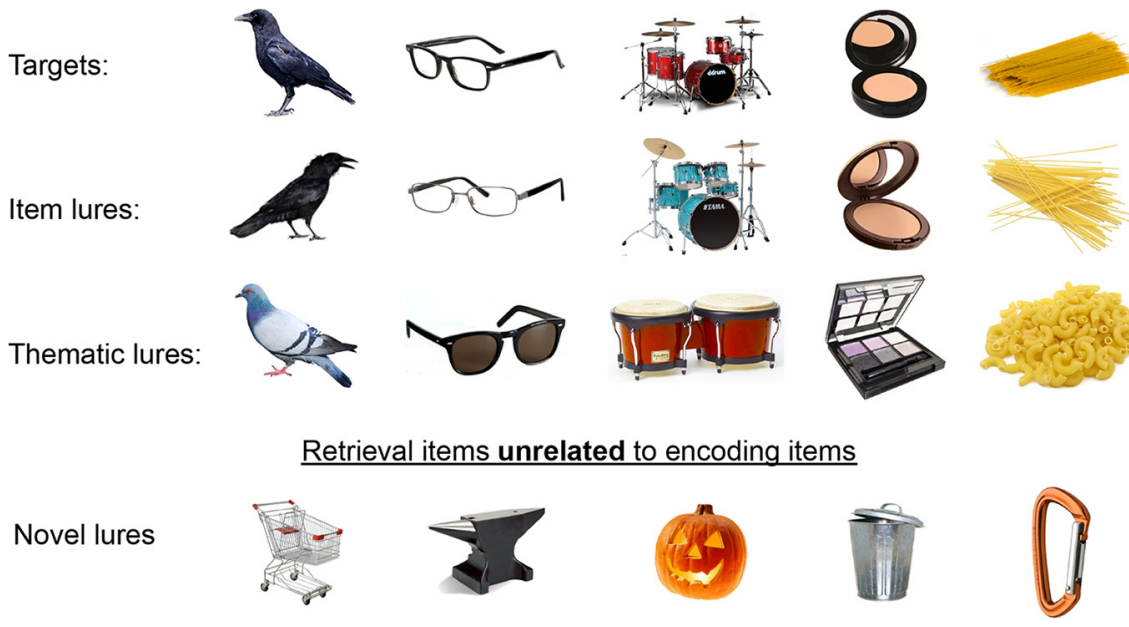

Figure 1. Example retrieval stimuli. During retrieval, targets (exact image from encoding) were presented as well as three types of lures: item lures (different image/exemplar of an item from encoding), thematic lures (item from the same higher order category or theme as an item from encoding), and completely novel lures not part of any category from encoding.

\section{Experimental design}

Participants completed the encoding and retrieval phases in a single scanning session. Stimuli were displayed using COGENT in MATLAB (The MathWorks) and were back-projected onto a screen that participants viewed through a mirror attached to the head coil. Behavioral responses were recorded using a four-button response box. Scanner noise was reduced with headphones and cushioning was used to reduce head motion.

Encoding was incidental and participants were instructed to make a size judgment for each of 96 items (i.e., "Is this item bigger or smaller than a shoebox?"). Each image was presented for $1500 \mathrm{~ms}$, followed by $500 \mathrm{~ms}$ additional responding time before automatically advancing to the next item. Each item was followed by a variable intertrial fixation $(\mathrm{M}=$ $2470 \mathrm{~ms}, \mathrm{SD}=1760 \mathrm{~ms}$ ) to aid in deconvolving the hemodynamic response (Dale, 1999). After the encoding phase, participants underwent structural scans and were given instructions for retrieval. During retrieval, participants were presented with all 96 studied items (targets), both the item and thematic lures from each of the encoding categories ( 96 of each) and 28 novel lures. All stimulus categories were presented in an intermixed fashion during each run pseudorandomly ordered to ensure that no more than three stimuli of the same type were presented sequentially. Each stimulus was displayed for $3 \mathrm{~s}$ while participants made their memory responses. Each trial was followed by a variable intertrial interval $(\mathrm{M}=2340 \mathrm{~ms}, \mathrm{SD}=1440 \mathrm{~ms})$. Consistent with typical instructions for the remember-know-new paradigm, participants were instructed to respond "remember" if they believed that the item was presented during the first phase of the experiment and they remembered specific, vivid details of its prior presentation. Participants were instructed to respond "familiar" if they believed the item was presented during the first phase but they could not recall specific details about its prior presentation. Instead of the typical "new" response, participants were asked to respond with two distinct "new" options: "unfamiliar" or "different" (Matzen et al., 2011). Participants were instructed to respond "different" if they believed the item was new and they could recall aspects of truly presented item(s) that indicated the item was not presented during the study phase (i.e., recollection rejection). Participants were instructed to respond "unfamiliar" if they believed an item was new because it did not resemble or bring to mind anything from the previous encoding phase (i.e., unfamiliar item). A practice session before the retrieval scans ensured that participants understood the retrieval instructions and the distinction between each response option before beginning the retrieval scans. Following scanning, participants completed a debriefing questionnaire that asked about their strategies during the task.

\section{fMRI data acquisition}

Images were acquired using a Siemens $3 \mathrm{~T}$ scanner equipped with a 12-channel head coil. A T1-weighted sagittal localizer was acquired to locate the AC-PC. Images were then prescribed at an oblique angle that prevented data collection in the area of the orbits. A highresolution anatomical image was acquired in the sagittal plane using a magnetization prepared rapid gradient echo sequence with a 1650 ms repetition time, $2.03 \mathrm{~ms}$ echo time, $256 \mathrm{~mm}$ field of view, $256 \mathrm{~mm}^{2}$ matrix, 160 sagittal slices, and $1 \mathrm{~mm}$ slice thickness for each participant. Echoplanar functional images were acquired using a descending acquisition, $2500 \mathrm{~ms}$ repetition time, $25 \mathrm{~ms}$ echo time, $240 \mathrm{~mm}$ field of view, an $80 \mathrm{~mm}^{2}$ matrix, and 42 axial slices with $3 \mathrm{~mm}$ slice thickness, resulting in $3 \mathrm{~mm}$ isotropic voxels. Ninety-one volumes were collected in each of two functional runs of the encoding task. One hundred seventy-five volumes were collected in each of the four functional runs of the retrieval task.

\section{Statistical analysis}

Behavioral data. To test for overall differences in memory performance across age groups, $d^{\prime}$ was calculated separately for young and older adults, collapsing across both types of old responses ("remember" and "know"), both types of new responses ("different" and "unfamiliar"), and including all lures (item, theme, and unrelated). These $d^{\prime}$ values were then submitted to an independent-samples $t$ test to test for age differences in memory discriminability. To further probe whether resulting age differences were due to changes in accuracy in endorsing old items or in rejecting lures, we computed a mixed-factor ANOVA with trial type (target, item lure, theme lure, novel lure) as a within-subject factor and age (young, older) as a between-subject factor and proportion labeled "old" (hit or false alarm) as the dependent variable.

fMRI preprocessing. Anatomical and functional images were skull stripped using the Brain Extraction Tool (BET) in FSL version 5.0.9 (www.fmrib.ox.ac.uk/fsl). FSL's MCFLIRT function was then used for within-run realignment and motion correction in each functional run, aligning all volumes to the middle volume. Between-run realignment was performed using Advanced Normalization Tools (ANTs) (http://stnava. github.io/ANTs/). The first volume of each run was aligned to the first volume of the first run (i.e., the first volume from the first encoding run) and that transformation was then applied to all volumes in the corresponding run. These realigned functional images were then submitted to FSL's fMRI Expert Analysis Tool (FEAT), where they were high-pass filtered at $100 \mathrm{~s}$ and underwent minimal spatial smoothing at $2 \mathrm{~mm}$ FWHM.

ROIs. To define subject-specific anatomical ROIs in native space, we submitted the high-resolution anatomical image from each subject to Freesurfer version 6 (https://surfer.nmr.mgh.harvard.edu/). Based on our interest in how age-related dedifferentiation in visual perceptual regions might relate to age deficits in memory specificity, we selected the following anatomical ROIs within the ventral visual cortex: midline occipital cortex (combination of lingual gyrus and cuneus labels in Freesurfer), lateral occipital cortex, posterior fusiform cortex (defined as the portion of fusiform cortex posterior to the most anterior slice of parahippocampal cortex), and inferotemporal cortex (Fig. 2). All ROIs were collapsed across hemispheres because we did not have hypotheses about lateralization in any region.

\section{fMRI analyses}

Univariate analyses. To account for potential differences in univariate activity across trial types that could drive classification effects, we generated a univariate model and included the resulting betas parameters as nuisance regressors in subsequent classification analyses (described below). A general linear model (GLM) was generated for the retrieval data using FSL'S FEAT with four regressors corresponding to onsets of test 


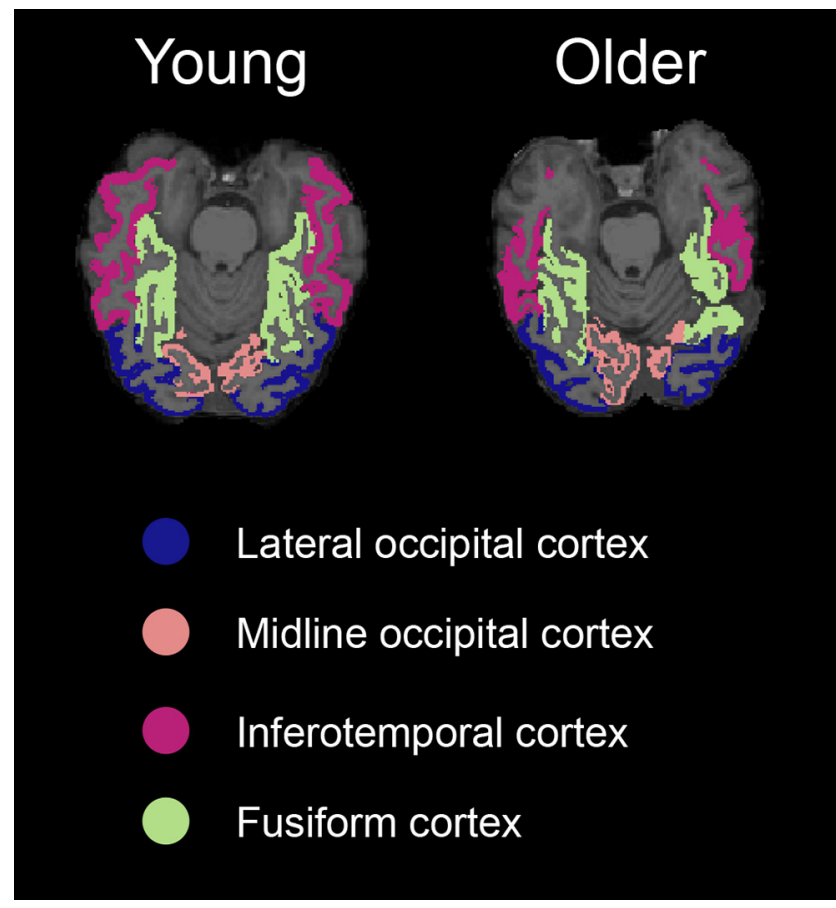

Figure 2. ROls. Four visual ROls were defined in native space using Freesurfer segmentation. We display the four regions as defined in a representative young adult and a representative older adult.

trial types: targets, item lures, thematic lures, and novel lures. To account for potential differences in reaction times across trial types and/or age groups, we included trial-by-trial reaction time as the duration of each trial. For trials in which participants failed to respond, reaction time was set to the full trial duration $(3 \mathrm{~s})$. An additional six nuisance regressors were included in each run, corresponding to translational and rotational movement. Mean $\beta$-parameters across each ROI were extracted for each run for each trial type of interest (targets, item lures, thematic lures) and then averaged across runs.

Multivariate pattern analyses. To estimate neural patterns associated with individual trials, a second GLM was generated using FSL's FEAT with one regressor for each trial at retrieval (79 regressors for each retrieval run). As above, trial-by-trial reaction time was included as the trial duration. An additional six nuisance regressors were included in each run corresponding to translational and rotational movement. From these models, individual whole-brain $\beta$ parameter maps were generated for each trial at retrieval. In a given parameter map, the value in each voxel represented the regression coefficient for that trial's regressor in a multiple regression containing all other trials in the run and the motion parameters. These $\beta$ parameter maps were then concatenated across runs and submitted to the PyMVPA toolbox (www.pymvpa.org) in Python (www.python.org) for pattern classification analyses.

\section{Pattern classification}

We were interested in which regions could distinguish between retrieval trial types that were related to information presented during study (i.e., targets, item lures, and thematic lures) and how age might change the neural discriminability of these trial types. Therefore, we computed separate classification accuracies for distinguishing between targets and item lures and targets and thematic lures, including all trials regardless of response accuracy. We computed separate two-way classifications rather than a single three-way classification so we could specify which targetlure distinctions were represented in a given region because abovechance three-way classification could occur if neural patterns for thematic lures were distinguishable from targets and items lures, but the latter were not distinguishable from one another. Novel lures were excluded from these analyses because rejection of novel lures is not typically affected by age. Further, there were fewer novel lures at retrieval, whereas the number of targets, item lures, and thematic lures were matched. Matching the number of trials across the three types of related trials allowed for training of the classifier without the classifier developing a bias toward a more common trial type.

Classification analyses were computed for the retrieval runs using a support vector machines (SVMs) classifier with a linear kernel using information from all voxels in each ROI. Training and testing followed a standard leave-one-run-out cross-validation procedure with three runs used as training data and one run as testing data. All possible train data/ test data permutations were computed for a given subject and the results averaged across validation folds. To test whether a given ROI carried information about the retrieval trial type, we computed one-tailed, onesample $t$ tests comparing classification accuracy to chance (50\%) for each of the comparisons. These initial $t$ tests were computed across age groups. In regions showing above-chance classification for a given comparison (target-thematic lure, target-item lure), we computed follow-up comparisons between age groups by submitting classification accuracy to an ANOVA that included age group as the only predictor of interest. To ensure that any effects identified were not driven by differences in overall motion across age groups or differences in univariate activity across age groups or trial types, we included covariates for average motion during retrieval for each translational and rotational direction (six covariates) and average univariate retrieval activity in a given ROI for each condition included in the particular classification analysis (i.e., targets and thematic lures or targets and item lures), totaling eight covariates in each ANOVA.

To assess whether classification was better for some types of comparisons than others and whether age affected the ability to distinguish between retrieval trial types, we submitted these classification accuracies to separate 2 (trial types compared: target-item lure, target-thematic lure) $\times 2$ (age group: young, older) mixed-factors ANOVAs for each ROI. As above, we included univariate retrieval activity (for targets, item lures, and thematic lures) in a given ROI and average subject motion (in six directions) as nuisance covariates, totaling nine covariates in each ANOVA.

To assess how the ability to classify brain patterns relates to behavioral performance, we computed separate multiple regression models for each ROI with overall behavioral $d^{\prime}$ as the dependent measure. We used a global measure of memory success ( $d^{\prime}$ including all three lure types) so that we could assess how the accuracy of each type of neural discrimination contributed to overall task performance, including the relative importance of each type of classification when they are included in the same model. Although we considered using separate $d^{\prime}$ measures for item and thematic lures, these two measures were highly correlated across subjects $(r=0.78, p<0.001)$ and thus likely to be driven by overlapping processes. Each regression analysis included the following predictors of interest: (1) both of the classification accuracies for the given ROI (target-item, target-thematic), (2) age group (young dummy coded as 0 , older dummy coded as 1) as a categorical predictor, and (3) an age $X$ classification accuracy interaction effect for each of the classification accuracies. We also included the above-described motion and univariate activity as nuisance regressors. To clarify the nature of any significant age interaction effect, we ran separate regressions in each age group and report the slopes separately for young and older adults. The model intercept was not of interest, but we report it in Table 4 for completeness. We did not mean center continuous predictors so that the interpretation of the intercept would be the same across all ROIs: the expected $d^{\prime}$ value in the young adult group if classification accuracies (target-item lure, target-thematic lure), all movement parameters, and all univariate predictors were zero.

To test whether target-lure discrimination was supported by any regions outside of the ventral visual cortex, we also computed a wholebrain searchlight analysis separately for target-item lure and target-thematic lure discriminations. The searchlight procedure followed that of the classification procedure within anatomical ROIs. An SVM classifier with a linear kernel was trained in a subject's native space within a gray matter mask using three runs of retrieval data and tested on the left-out run. All permutations of train-data/test-data were used and classification was averaged across validation folds. The searchlight was performed using a sphere with a $6 \mathrm{~mm}$ radius, generating a whole-brain map in each subject's native space depicting the classification accuracy when the sphere was centered over a 
Table 2. Retrieval response rates for each age group

\begin{tabular}{lll}
\hline & \multicolumn{1}{l}{ Young } & Older \\
\hline Targets & & \\
Remember & $0.59(0.15)$ & $0.59(0.17)$ \\
Familiar & $0.21(0.12)$ & $0.23(0.13)$ \\
Different & $0.06(0.05)$ & $0.05(0.07)$ \\
Unfamiliar & $0.14(0.07)$ & $0.12(0.07)$ \\
Item lures & & \\
Remember & $0.10(0.07)$ & $0.24(0.12)$ \\
Familiar & $0.18(0.16)$ & $0.29(0.16)$ \\
Different & $0.59(0.19)$ & $0.33(0.20)$ \\
Unfamiliar & $0.13(0.07)$ & $0.13(0.09)$ \\
Thematic lures & & $0.03(0.04)$ \\
Remember & $0.01(0.02)$ & $0.09(0.08)$ \\
Familiar & $0.05(0.08)$ & $0.28(0.14)$ \\
Different & $0.30(0.15)$ & $0.59(0.15)$ \\
Unfamiliar & $0.63(0.18)$ & \\
Novel lures & & $0.003(0.01)$ \\
Remember & $0.001(0.01)$ & $0.01(0.03)$ \\
Familiar & $0.01(0.02)$ & $0.04(0.07)$ \\
Different & $0.05(0.05)$ & $0.94(0.08)$ \\
Unfamiliar & $0.92(0.07)$ & $50000)$ \\
\hline
\end{tabular}

Data are shown as means (SD) of response rates for each trial type and each retrieval response separated by age group.

given voxel. To compare searchlight results across individuals and across groups, native space maps were warped into standard MNI space using ANTs. We first calculated the rigid affine transformation from the reference functional image (first volume from the first encoding run) to the T1 anatomical image and the nonlinear transformation of the $\mathrm{T} 1$ anatomical image to MNI space. We then applied both transformations to the classification maps, resulting in whole-brain classification maps in MNI space.

Group-level statistics of searchlight analyses were computed using FSL's Randomise function (Winkler et al., 2014). One-sample $t$ tests were computed for each classification (target-item, target-thematic) across age groups to determine regions that showed overall above-chance classification. To define an initial cluster-forming threshold, we used threshold-free cluster enhancement within a gray matter mask (defined from the Harvard Cortical and Subcortical atlases thresholded at a probability of $15 \%$ ) and using variance smoothing of $2 \mathrm{~mm}$. The resulting map was used to threshold the one-sample $t$-statistic map with voxels showing evidence of clustering at $p<0.05$. We applied an additional $t$-statistic threshold of $t>3.3(p<0.001)$ and a minimum cluster extent of 10 voxels. To identify regions showing both above-chance classification and significant age differences, we used 5000 iterations of the twosample $t$ test procedure in Randomise to generate four $t$-test contrasts: young adults $>$ chance, young adults $>$ older adults, older adults $>$ chance, and older adults $>$ young adults. These $t$ tests included subjectwise motion parameters as covariates, but not univariate activation given that univariate activation would vary for every voxel. We used a $t$-statistic threshold of $t=1.7(p<0.05)$ for the group differences, masking with the appropriate age group $>$ chance contrast thresholded as described above. For example, the young adult $>$ older adult contrast was inclusively masked with the young $>$ chance contrast. This procedure ensured that significant age differences were not driven by differences in at- or belowchance performance in both groups. Regions showing significant effects were labeled using the Harvard-Oxford cortical and subcortical atlases as implemented in FSL (Desikan et al., 2006).

For all analyses, a Greenhouse-Geisser correction was used to account for violations of sphericity where appropriate. In addition, we corrected for multiple comparisons when computing separate tests across the four ROIs using the Bonferroni method. For regression analyses, we corrected the $p$-value for the overall regression model, but present uncorrected $p$-values for individual predictors given that the multiple regression framework requires individual effects to be significant above and beyond any other effects included in the model. Corrected $p$-values for all other effects are presented in the Results section below and in the tables.
A

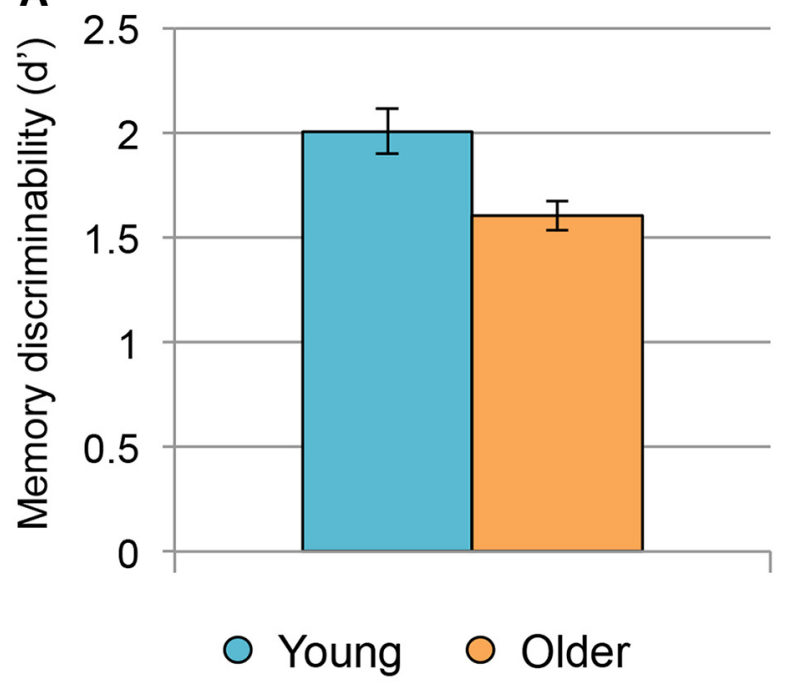

B

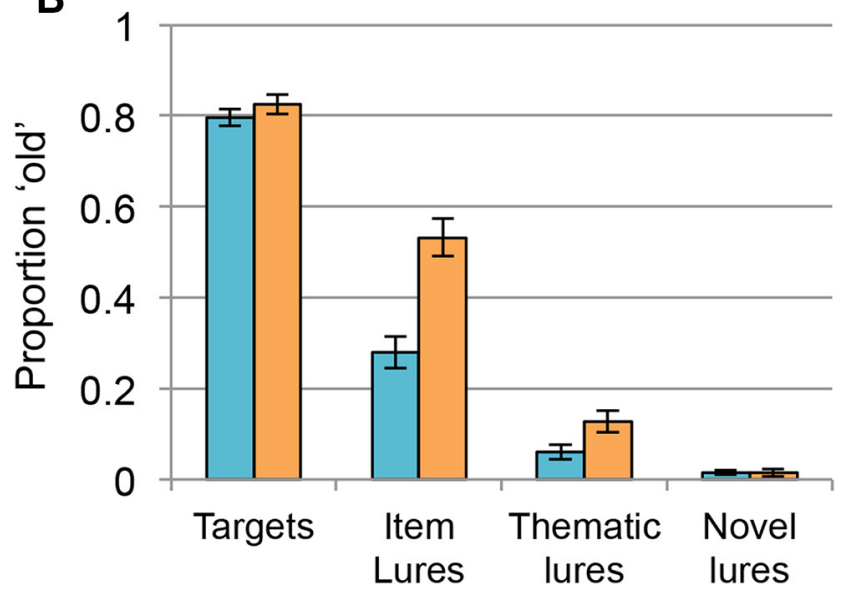

Figure 3. Behavioral accuracy at retrieval. $A$, Young adults (blue) showed significantly better memory discriminability compared with older adults (orange) as measured by an overall $d^{\prime}$ value. $\boldsymbol{B}$, Although young and older adults endorsed targets as old at similar rates, older adults showed significantly higher rates of false recognition for item and thematic, but not novel, lures. Error bars indicate SEM across subjects.

\section{Results}

\section{Behavioral data}

Means and SDs of retrieval response rates separated by age group are reported in Table 2. Overall memory performance was indexed by $d^{\prime}$ collapsed across lure types and across multiple old ("remember," "familiar") and new ("different," "unfamiliar") responses. As expected, young adults showed significantly higher $d^{\prime}$ than older adults $\left(t_{(46)}=3.10, p=0.003, d=0.90\right.$; Fig. $3 A$ ), indicating that older adults did not distinguish between old and new items during retrieval as well as young adults.

We were also interested in whether this overall age deficit was driven by older adults' greater difficulty in rejecting lures at retrieval. We therefore computed a mixed-factor ANOVA with age and trial type as factors and proportion of items labeled "old" as the dependent variable (Fig. $3 B$ ). Results revealed a significant main effect of trial type $\left(F_{(1.83,84.15)}=708.94, p<0.001\right.$, partial $\left.\eta^{2}=0.94\right)$ with the highest proportion of endorsement for targets $(M=0.81, S D=0.10)$, followed by item lures $(M=0.41$, $\mathrm{SD}=0.19)$, then thematic lures $(M=0.10, \mathrm{SD}=0.10)$, and the lowest endorsement of novel lures $(\mathrm{M}=0.02, \mathrm{SD}=0.03)$. There was also a significant main effect of age $\left(F_{(1,46)}=12.66, p=0.001\right.$, 
partial $\left.\eta^{2}=0.22\right)$, with older adults endorsing items as old at higher rates $(\mathrm{M}=0.38, \mathrm{SD}=0.09)$ than young adults $(\mathrm{M}=0.29$, $\mathrm{SD}=0.09)$. Finally, there was a significant age $\times$ trial type interaction age $\left(F_{(1.83,84.15)}=17.80, p<0.001\right.$, partial $\left.\eta^{2}=0.28\right)$. Post hoc independent-samples $t$ tests showed that older adults had more false recognitions than young adults for item lures $\left(t_{(46)}\right.$ $=4.72, p<0.001, d=0.96)$ and thematic lures $\left(t_{(46)}=2.32, p=\right.$ $0.03, d=0.47)$, but that reliable age differences did not emerge for targets $\left(t_{(46)}=1.01, p=0.32, d=0.21\right)$ or novel lures $\left(t_{(46)}=\right.$ $0.63, p=0.53, d=0.04)$. In addition, to confirm that the observed age difference in false recognition was larger for item lures than for thematic lures, we ran a 2 (age group: young, older) $\times 2$ (lure type: item lure, thematic lure) mixed-factor ANOVA and confirmed that the age $\times$ lure type interaction was indeed significant $\left(F_{(1,46)}=23.86, p<0.001\right.$, partial $\left.\eta^{2}=0.34\right)$.

\section{fMRI data}

Accuracy of pattern classification: anatomical ROIs

We first compared classification accuracy for targets versus thematic lures to theoretical chance (proportion correct $=0.5$ ) in each ROI collapsed across age groups. Results revealed abovechance performance in each ROI: midline occipital cortex $\left(t_{(47)}=8.32, p<0.001, d=1.20\right)$, lateral occipital cortex $\left(t_{(47)}=\right.$ 4.97, $p<0.001, d=0.72)$, fusiform cortex $\left(t_{(47)}=4.30, p<\right.$ $0.001, d=0.62)$, and inferotemporal cortex $\left(t_{(47)}=6.56, p<\right.$ $0.001, d=0.95)$. One-sample $t$ tests comparing target-item lure classification to chance showed that the midline occipital ROI distinguished between these highly similar item types at abovechance levels $\left(t_{(47)}=3.82, p<0.001, d=0.55\right)$, but no other ROI made this distinction: lateral occipital cortex $\left(t_{(47)}=1.02, p=\right.$ $0.31, d=0.15)$, fusiform cortex $\left(t_{(47)}=1.15, p=0.26, d=0.17\right)$, and inferotemporal cortex $\left(t_{(47)}=1.11, p=0.27, d=0.16\right)$. Mean classification accuracy for each age group in each ROI for target-thematic lure discrimination is presented in Figure $4 A$ and for target-item lure discrimination in Figure $4 B$.

In addition to identifying regions showing signals for targets that were discriminable from those of lures, we also tested whether there were reliable age differences in those regions. In each region showing above-chance classification performance, we computed a separate ANOVA comparing young and older adults, including univariate activation and motion parameters as nuisance covariates. None of the ROIs showed a significant age difference for target-thematic lure classification (midline occipital cortex: $F_{(1,38)}=2.23, p=0.56$, partial $\eta^{2}=0.06$; lateral occipital cortex: $F_{(1,38)}=0.09, p=1.00$, partial $\eta^{2}=0.002$; fusiform cortex: $F_{(1,38)}=1.45, p=0.96$, partial $\eta^{2}=0.04$; inferotemporal cortex: $F_{(1,38)}=0.59, p=1.00$, partial $\left.\eta^{2}=0.02\right)$. However, the midline occipital cortex, which was the only ROI to show above chance target-item lure classification, did reveal a significant age deficit $\left(F_{(1,38)}=6.56, p=0.015\right.$, partial $\left.\eta^{2}=0.15\right)$.

Last, to test for differences in accuracy for target-thematic versus target-item lure classification and whether age moderated any potential differences, we computed a 2 (age: young, older) $\times$ 2 (trial type comparison: target-thematic, target-item) mixedfactors ANOVA in each ROI while controlling for subject motion and univariate activation. Results for each ANOVA are presented in Table 3. We found that target-thematic lure classification was significantly higher than target-item lure classification only in midline occipital cortex, but that age group did not significantly moderate this difference. No other effects reached significance once corrected for multiple comparisons (all corrected $p>0.15$ ). Therefore, although there was a significant age deficit in target-

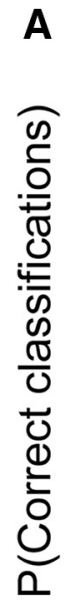
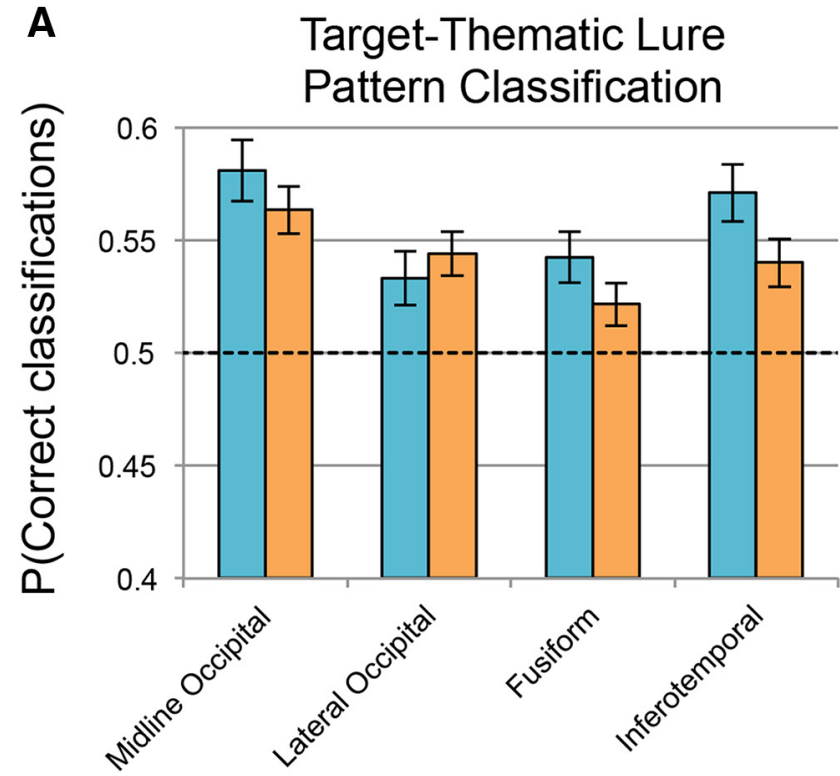

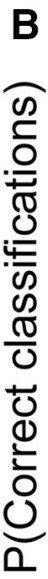
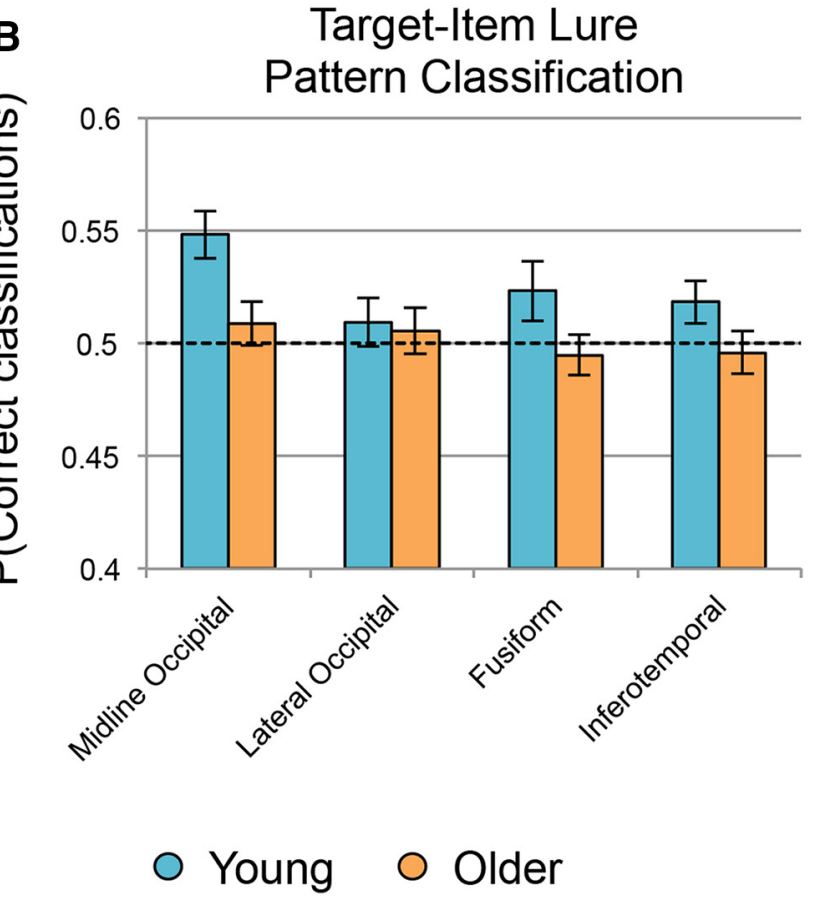

Figure 4. Pattern classification analyses. Shown is the mean accuracy for a classifier trained to distinguish between targets and thematic lures $(\boldsymbol{A})$ and targets and item lures $(\boldsymbol{B})$. Results are presented across four anatomically defined ROls in ventral visual cortex in young (blue) and older adults (orange). Dashed line represents theoretical chance and error bars indicate SEM across subjects.

item lure classification performance in midline occipital cortex, that age deficit was only numerically larger than the (nonsignificant) age deficit for target-thematic lure classification.

\section{Relationship between pattern classification accuracy and memory discriminability}

To measure how neural discriminability between targets and lures related to behavioral discriminability, we computed a multiple regression in each ROI with behavioral $d^{\prime}$ as the dependent variable and controlling for subject motion and univariate activation. Table 4 includes the $\beta$-, $t$-, and $p$-values for model parameters of interest in each ROI. 
Table 3. Comparisons between target-thematic lure and target-item lure classification

\begin{tabular}{lllll}
\hline Predictor & $\mathrm{df}$ & $F$ & $p$ & $\eta^{2}$ \\
\hline ROI: Midline occipital cortex & & & & \\
$\quad$ Age & 1,37 & 4.66 & 0.16 & 0.11 \\
$\quad$ Trial comparison* & 1,37 & 9.56 & 0.02 & 0.21 \\
$\quad$ Trial comparison $\times$ age & 1,37 & 0.89 & 1.00 & 0.02 \\
ROI: Lateral occiptial cortex & & & & \\
$\quad$ Age & 1,37 & 0.04 & 1.00 & 0.001 \\
$\quad$ Trial comparison & 1,37 & 0.04 & 1.00 & 0.001 \\
$\quad$ Trial comparison $\times$ age & 1,37 & 0.05 & 1.00 & 0.001 \\
ROI: Fusiform cortex & & & & \\
$\quad$ Age & 1,37 & 2.04 & 0.64 & 0.05 \\
$\quad$ Trial comparison & 1,37 & 0.41 & 1.00 & 0.01 \\
$\quad$ Trial comparison $\times$ age & 1,37 & 0.01 & 1.00 & $<0.001$ \\
ROI: Inferotemporal cortex & & & & \\
$\quad$ Age & 1,37 & 0.90 & 1.00 & 0.02 \\
$\quad$ Trial comparison & 1,37 & 1.08 & 1.00 & 0.03 \\
$\quad$ Trial comparison $\times$ age & 1,37 & 0.03 & 1.00 & 0.001 \\
\hline
\end{tabular}

For each ROl, results from a 2 (age: young, older) $\times 2$ (trial type comparison: targets-thematic lures, targets-item lures) mixed-factors ANOVA, including two main effects and one interaction effect.

$\mathrm{df}$, Degrees of freedom; $F=F$-statistic; $\eta^{2}=$ partial $\eta^{2}$.

*Effects significant after correcting for multiple comparisons.

In the midline occipital ROI, the overall model predicting memory discriminability was significant $\left(F_{(14,33)}=3.13, p=\right.$ $\left.0.01, R^{2}=0.57\right)$, with only the target-item lure classification accuracy predictor reaching significance. Therefore, across age groups, better discrimination of targets and item lures in midline occipital regions was associated with better behavioral discriminability. The overall model also reached significance in the lateral occipital ROI $\left(F_{(14,33)}=3.48, p=0.006, R^{2}=0.60\right)$, with age group, target-thematic lure classification, and the target-thematic lure classification $\times$ age interaction predictors all reaching significance. The negative parameter estimate for the interaction reflects that young adults showed a positive relationship between target-thematic lure classification and memory discrimination $(\beta=0.59, t=3.79, p=0.01)$ whereas older adults showed a numerically negative relationship $(\beta=-0.38, t=-1.42$, $p=$ $0.18)$. A similar pattern emerged in the fusiform cortex $\left(F_{(14,33)}=\right.$ 4.06, $p<0.002, R^{2}=0.63$ ) with the age group, target-thematic lure classification, and target-thematic lure classification $\times$ age predictors all reaching significance and the interaction effect reflecting a marginally positive relationship in young adults $(\beta=$ $0.33, t=2.15, p=0.05)$ and a negative relationship in older adults $(\beta=-0.56, t=-2.82, p=0.04)$. Finally, the model was also significant in inferotemporal cortex $\left(F_{(14,33)}=3.45, p=\right.$ $\left.0.007, R^{2}=0.59\right)$, in which the target-thematic lure classification accuracy predictor was significant, but the target-thematic lure classification $\times$ age interaction was only marginally significant (corrected $p=0.05$ ) despite the overall pattern being similar to effects in lateral occipital and fusiform cortex.

Accuracy of pattern classification: whole-brain searchlight

Although we found reliable target-item lure discrimination only in midline occipital cortex, we were interested in whether regions outside of the ventral visual stream also contributed to the discriminability of highly similar items in memory. In addition, although we found reliable target-thematic lure decoding throughout ventral visual cortex, we were interested in whether this strong decoding was consistent across the rest of the brain. To test for effects outside of the visual cortex, we computed a whole-brain searchlight analysis separately for target-thematic lures and target-item lures.
Table 4. Relationship between neural discriminability and behavioral discriminability

\begin{tabular}{lrrl}
\hline Predictor & \multicolumn{1}{l}{$l$} & $p$ \\
\hline ROI: Midline occipital cortex & & & \\
Intercept & -1.22 & -1.36 & 0.18 \\
Age & 1.61 & 0.87 & 0.39 \\
Target-thematic lure & 0.14 & 0.70 & 0.49 \\
Target-item lure* & 0.52 & 2.39 & 0.02 \\
Target-thematic lure $\times$ age & 0.08 & 0.06 & 0.96 \\
Target-item lure $\times$ age & -1.73 & -1.18 & 0.25 \\
ROI: Lateral occipital cortex & & & \\
Intercept & -0.29 & -0.27 & 0.79 \\
Age* & 4.17 & 2.50 & 0.02 \\
Target-thematic lure* & 0.57 & 3.80 & 0.001 \\
Target-item lure & -0.12 & -0.73 & 0.47 \\
Target-thematic lure $\times$ age* & -5.00 & -3.66 & 0.001 \\
Target-item lure $\times$ age & 0.47 & 0.38 & 0.71 \\
Rol: Fusiform cortex & & & \\
Intercept & -1.28 & -1.48 & 0.15 \\
Age* & 5.83 & 3.88 & $<0.001$ \\
Target-thematic lure* & 0.47 & 3.14 & 0.004 \\
Target-item lure & 0.18 & 1.24 & 0.23 \\
Target-thematic lure $\times$ age & -4.44 & -3.44 & 0.002 \\
Target-item lure $\times$ age & -1.55 & -1.24 & 0.22 \\
ROI: Inferotemporal cortex & & & \\
Intercept & -0.01 & -0.01 & 0.99 \\
Age & -0.27 & -0.16 & 0.88 \\
Target-thematic lure* & 0.57 & 3.69 & 0.001 \\
Target-item lure & -0.15 & -0.86 & 0.40 \\
Target-thematic lure $\times$ age & -2.69 & -2.05 & 0.05 \\
Target-item lure $\times$ age & 2.81 & 1.76 & 0.09 \\
\hline
\end{tabular}

For each ROI, results from a regression including age group (coded as young $=0$, older $=1$ ), target-thematic lure classification, target-item lure classification, and their interactions as predictors of overall $d^{\prime}$ values. Average univariate activation for targets, item lures, and thematic lures as well as average subject-wise motion were also included as nuisance covariates.

$\beta$, Standardized beta parameter; $t, t$-statistic of peak voxels.

*Effects significant at $p<0.05$.

Regions where target-thematic lure classification accuracy was above chance across the entire group regardless of age are listed in the upper portion of Table 5. This contrast revealed a broad map of regions with signals that distinguished between these two trial types, including one large cluster that spanned bilateral cortical and subcortical regions (Fig. 5A). Regions where target-thematic lure classification was significantly better for young adults compared with older adults are listed in the lower portion of Table 5 and depicted in Figure $5 B$. There were no regions where target-thematic lure classification accuracy was better in older compared with young adults.

Regions where target-item lure classification accuracy was above chance across the entire group regardless of age are listed in the upper portion of Table 6. Consistent with the ROI analyses, portions of midline occipital cortex (lingual gyrus, cuneus) had signals that could distinguish between these similar trial types in addition to several other cortical regions (Fig. 5C). Regions where target-item lure classification was significantly better for young adults compared with older adults are listed in the lower portion of Table 6 and depicted in Figure 5D. There were no regions where target-item lure classification accuracy was better in older compared with young adults.

\section{Discussion}

Aging reduces the specificity of visual representations underlying memory decisions, but the degree of specificity decline has remained an open question. We tested whether patterns of activation in ventral visual cortex could be used to discriminate 
Table 5. Whole-brain searchlight of target-thematic lure classification

\begin{tabular}{|c|c|c|c|c|c|c|}
\hline Region & $\mathrm{H}$ & k & $t$ & $x$ & $y$ & $z$ \\
\hline \multicolumn{7}{|c|}{ Target-thematic lure classification: whole group $>$ theoretical chance } \\
\hline Multiple cortical and subcortical regions & B & 62597 & 8.83 & 4 & -52 & 26 \\
\hline Temporal pole & $\mathrm{R}$ & 11 & 4.01 & 40 & 20 & -26 \\
\hline Caudate & $\mathrm{L}$ & 69 & 5.23 & -12 & 12 & -2 \\
\hline \multirow[t]{2}{*}{ Parahippocampal cortex } & $\mathrm{L}$ & 11 & 3.84 & -20 & 0 & -22 \\
\hline & $\mathrm{R}$ & 10 & 3.89 & 22 & -30 & -20 \\
\hline Hippocampus & $\mathrm{R}$ & 19 & 4.01 & 32 & -36 & -2 \\
\hline \multirow[t]{2}{*}{ Cingulate cortex } & M & 10 & 4.55 & 4 & -8 & 36 \\
\hline & $\mathrm{L}$ & 19 & 4.42 & -10 & -10 & 46 \\
\hline Inferotemporal cortex & $\mathrm{L}$ & 14 & 4.5 & -54 & -10 & -38 \\
\hline Occipital pole & M & 11 & 4.04 & -6 & -100 & 8 \\
\hline \multicolumn{7}{|c|}{ Target-thematic lure classification: young $>$ older adults } \\
\hline \multirow[t]{5}{*}{ Frontal pole } & $\mathrm{L}$ & 11 & 3.42 & -38 & 58 & -10 \\
\hline & $\mathrm{L}$ & 278 & 4.21 & -46 & 38 & 16 \\
\hline & $\mathrm{R}$ & 11 & 2.33 & 24 & 62 & 2 \\
\hline & $\mathrm{R}$ & 47 & 3.47 & 24 & 58 & 16 \\
\hline & $\mathrm{R}$ & 31 & 2.85 & 22 & 54 & 38 \\
\hline \multirow[t]{6}{*}{ Superior frontal gyrus } & $\mathrm{L}$ & 20 & 3.17 & -20 & 38 & 52 \\
\hline & $\mathrm{L}$ & 23 & 2.49 & -26 & 22 & 58 \\
\hline & $\mathrm{L}$ & 24 & 3.53 & -22 & 8 & 64 \\
\hline & $\mathrm{L}$ & 72 & 3.24 & -24 & -10 & 48 \\
\hline & M & 246 & 3.62 & -2 & 50 & 42 \\
\hline & M & 12 & 3.3 & -4 & 38 & 52 \\
\hline \multirow[t]{5}{*}{ Middle frontal gyrus } & $\mathrm{L}$ & 69 & 3.18 & -44 & 32 & 30 \\
\hline & $\mathrm{L}$ & 29 & 2.9 & -40 & 28 & 44 \\
\hline & $\mathrm{L}$ & 20 & 2.81 & -40 & 14 & 56 \\
\hline & $\mathrm{L}$ & 20 & 3.09 & -34 & -4 & 58 \\
\hline & $\mathrm{R}$ & 671 & 4.07 & 44 & 28 & 42 \\
\hline \multirow[t]{5}{*}{ Inferior frontal gyrus } & $\mathrm{L}$ & 172 & 3.5 & -52 & 26 & 22 \\
\hline & $\mathrm{L}$ & 12 & 3.62 & -54 & 22 & 14 \\
\hline & $\mathrm{L}$ & 32 & 2.71 & -44 & 18 & -6 \\
\hline & $\mathrm{L}$ & 11 & 2.61 & -54 & 16 & 6 \\
\hline & $\mathrm{R}$ & 13 & 2.54 & 50 & -10 & 18 \\
\hline Dorsomedial prefrontal cortex & M & 64 & 3.14 & 4 & 50 & 24 \\
\hline Insular cortex & $\mathrm{L}$ & 89 & 3.11 & -44 & -4 & -10 \\
\hline \multirow[t]{5}{*}{ Precentral gyrus } & $\mathrm{L}$ & 25 & 3.1 & -48 & -14 & 62 \\
\hline & $\mathrm{L}$ & 14 & 3.28 & -34 & -20 & 44 \\
\hline & $\mathrm{R}$ & 17 & 2.48 & 60 & -4 & 28 \\
\hline & $\mathrm{R}$ & 16 & 2.6 & 34 & -6 & 64 \\
\hline & $\mathrm{R}$ & 11 & 2.34 & 38 & -8 & 50 \\
\hline \multirow[t]{2}{*}{ Postcentral gyrus } & $\mathrm{L}$ & 17 & 2.68 & -52 & -22 & 58 \\
\hline & $\mathrm{L}$ & 12 & 3.29 & -40 & -24 & 66 \\
\hline Superior temporal gyrus & $\mathrm{R}$ & 216 & 3.35 & 48 & -34 & 4 \\
\hline \multirow[t]{3}{*}{ Middle temporal gyrus } & $\mathrm{L}$ & 12 & 2.74 & -56 & -58 & -2 \\
\hline & $\mathrm{R}$ & 10 & 2.15 & 62 & -36 & -4 \\
\hline & $\mathrm{R}$ & 83 & 2.93 & 52 & -48 & -4 \\
\hline \multirow[t]{4}{*}{ Inferotemporal cortex } & $\mathrm{L}$ & 14 & 2.41 & -54 & -24 & -20 \\
\hline & $\mathrm{L}$ & 268 & 3.63 & -50 & -50 & -24 \\
\hline & $R$ & 10 & 3.32 & 34 & -46 & -6 \\
\hline & $\mathrm{R}$ & 16 & 2.59 & 54 & -56 & -12 \\
\hline \multirow[t]{2}{*}{ Posterior cingulate } & M & 17 & 3.27 & -4 & -34 & 34 \\
\hline & M & 13 & 2.84 & -4 & -46 & 38 \\
\hline \multirow[t]{5}{*}{ Precuneus } & $\mathrm{L}$ & 13 & 2.29 & -10 & -38 & 46 \\
\hline & $\mathrm{L}$ & 29 & 3.41 & -18 & -52 & 4 \\
\hline & $\mathrm{R}$ & 312 & 3.41 & 28 & -56 & 6 \\
\hline & $\mathrm{R}$ & 33 & 3.07 & 10 & -64 & 32 \\
\hline & $\mathrm{R}$ & 30 & 2.9 & 20 & -64 & 24 \\
\hline Superior parietal cortex & $R$ & 29 & 3.25 & 26 & -54 & 40 \\
\hline Angular gyrus & $\mathrm{L}$ & 88 & 3.51 & -60 & -60 & 20 \\
\hline & $\mathrm{R}$ & 14 & 2.32 & 62 & -48 & 34 \\
\hline Fusiform cortex & $\mathrm{L}$ & 14 & 3.25 & -36 & -70 & -10 \\
\hline & $\mathrm{R}$ & 14 & 2.89 & 30 & -72 & -6 \\
\hline
\end{tabular}

Table 5. Continued

\begin{tabular}{|c|c|c|c|c|c|c|}
\hline Region & $\mathrm{H}$ & $k$ & $t$ & $x$ & $y$ & $Z$ \\
\hline \multirow[t]{11}{*}{ Lateral occipital cortex } & $\mathrm{R}$ & 12 & 2.41 & 50 & -58 & 48 \\
\hline & $\mathrm{L}$ & 10 & 2.22 & -44 & -62 & 20 \\
\hline & $\mathrm{L}$ & 13 & 2.89 & -20 & -66 & 56 \\
\hline & $\mathrm{L}$ & 15 & 2.73 & -54 & -70 & -6 \\
\hline & $\mathrm{L}$ & 10 & 3.01 & -12 & -76 & 48 \\
\hline & $\mathrm{L}$ & 132 & 3.14 & -50 & -78 & 20 \\
\hline & $\mathrm{L}$ & 23 & 3.02 & -10 & -82 & 44 \\
\hline & $\mathrm{L}$ & 44 & 3.01 & -38 & -86 & 8 \\
\hline & $\mathrm{L}$ & 55 & 3.09 & -38 & -92 & -2 \\
\hline & $\mathrm{R}$ & 27 & 2.86 & 40 & -74 & -6 \\
\hline & $\mathrm{R}$ & 18 & 2.96 & 48 & -74 & 4 \\
\hline \multirow[t]{5}{*}{ Lingual gyrus } & $\mathrm{L}$ & 232 & 3.86 & -28 & -50 & -6 \\
\hline & $\mathrm{L}$ & 25 & 2.23 & -6 & -66 & 6 \\
\hline & $M$ & 46 & 3.72 & -2 & -72 & -2 \\
\hline & M & 10 & 2.77 & 4 & -82 & 0 \\
\hline & $\mathrm{R}$ & 84 & 3.43 & 22 & -60 & -12 \\
\hline Cuneus & $\mathrm{R}$ & 20 & 2.77 & 16 & -80 & 34 \\
\hline Calcarine cortex & $\mathrm{L}$ & 22 & 2.96 & -12 & -84 & 4 \\
\hline \multirow[t]{5}{*}{ Occipital pole } & $\mathrm{L}$ & 20 & 3.27 & -12 & -100 & 18 \\
\hline & $M$ & 10 & 2.68 & 8 & -94 & 20 \\
\hline & $M$ & 21 & 2.86 & -8 & -96 & 30 \\
\hline & $\mathrm{R}$ & 13 & 3.04 & 26 & -88 & 34 \\
\hline & $\mathrm{R}$ & 10 & 3.02 & 18 & -90 & 36 \\
\hline
\end{tabular}

$H$, Hemisphere; $k$, cluster size in number of voxels; $t, t$-statistic of peak voxel, $x, y, z$, peak coordinates in MNI space.

between old and new retrieval items at multiple levels of targetlure similarity and if age affected this neural discrimination. We found that targets and lures were discriminable from signals in all the ventral visual regions that we tested when lures differed from targets in both perceptual and semantic details (thematic lures). However, targets and lures were discriminable from activation patterns in midline occipital cortex even when lures differed from targets only in specific perceptual details (item lures), suggesting that the visual representations in this early visual region are highly detailed and contribute to making fine-grained memory discriminations. Although there was an overall age deficit in neural target-item lure discrimination, higher classification accuracy in midline occipital cortex was nonetheless associated with better memory performance across age groups. This result suggests that, whereas these representations are weaker overall in older compared with young adults, the fundamental nature of the information represented in these regions is unlikely to differ between age groups. Conversely, in lateral occipital and fusiform cortices, age moderated the relationship between neural targetthematic lure discrimination and behavioral discrimination, suggesting age differences in the types of representations maintained in these regions and brought to bear when making memory decisions.

Prior work has shown that ventral visual regions contribute to memory retrieval by reactivating visual details from a previous episode (Polyn et al., 2005; Watanabe et al., 2012). The strength of this reactivation is related to memory vividness (Wheeler et al., 2000; Johnson and Rugg, 2007; Buchsbaum et al., 2012) and to the accuracy of memory judgments (Kuhl et al., 2011; Wing et al., 2015). The present study builds on prior research by demonstrating that old/new differences are detectable from ventral visual signals across perceptually diverse stimuli, consistent with the idea that regions involved primarily with visual perception also contain memory signals (Schacter and Buckner, 1998; Henson and Rugg, 2003). Specifically, we found that targets and lures were distinguishable from signals across ventral visual cortex when they differed both in perceptual details and semantic labels 
A

Target-Thematic Lure Classification: Whole group $>$ chance

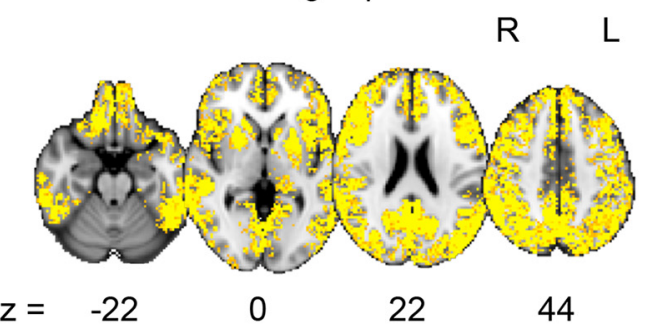

B

Target-Thematic Lure Classification: Young $>$ Older adults

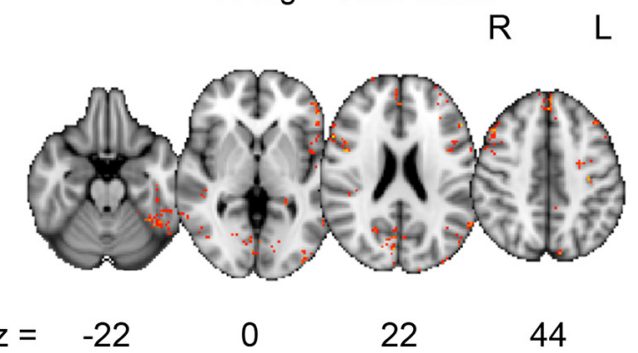

C

Target-Item Lure Classification: Whole group $>$ chance

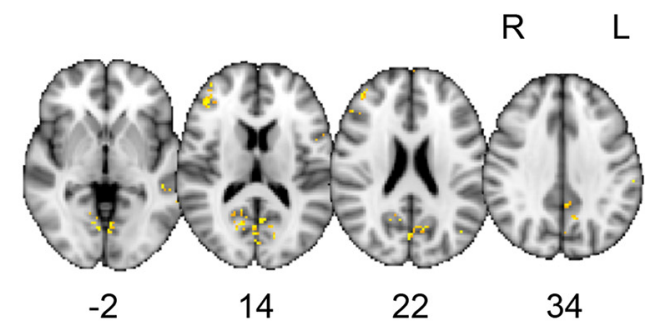

D

Target-Item Lure Classification: Young $>$ Older adults

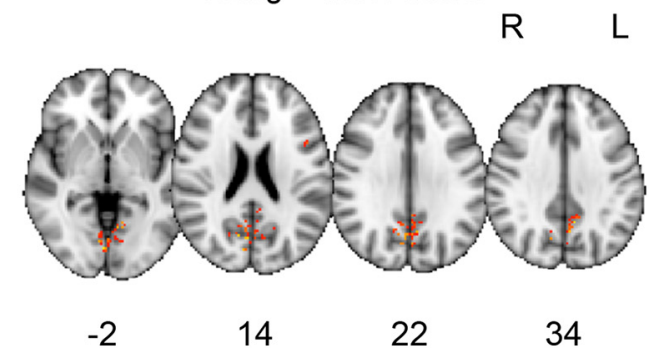

Figure 5. Whole-brain searchlight pattern classification analyses. Regions where target-thematic lure classification was above chance collapsed across age groups ( $\boldsymbol{A}$ ) and where classification accuracy was significantly higher in young compared with older adults $(\boldsymbol{B})$. Regions where target-item lure classification was above chance collapsed across age groups $(\boldsymbol{C})$ and where classification accuracy was significantly higher in young compared with older adults $(\boldsymbol{D})$. There were no regions where classification accuracy was significantly higher in older compared with young adults for either target-thematic lure or target-item lure classification.

(thematic lures). Conversely, we found that targets and lures were distinguishable only from signals in midline occipital cortex of our visual ROIs when targets and lures differed only in perceptual details (item lures). This finding is consistent with studies showing that early visual regions contain signals that distinguish between true and false memories (Slotnick and Schacter, 2004), particularly when true memories are associated with retrieval of vivid details from encoding (Dennis et al., 2012). We also found that older adults showed poorer neural discriminability of targets and item lures in this region, which complements previous studies showing reductions in early visual activation for true memories in older adults compared with young adults (Gutchess et al., 2005; Dennis et al., 2014). The current results add to this previous work by showing that, in addition to univariate reductions in early visual activity, neural patterns representing targets and item lures also become less distinguishable with increasing age.

Despite an age deficit in target-item lure discriminability in midline occipital cortex, analyses linking neural discrimination to behavioral memory discrimination showed a positive relationship across age groups. Therefore, similar types of information seem to be detectable across age groups even though the response distributions underlying this classification differ substantially between young and older adults. We propose that this positive brain-behavior relationship emerges because individuals who reactivate more specific perceptual details from a prior encoding event are better able to detect lure features that differ from targets, aiding in lure rejection particularly when lures strongly resemble targets (i.e., recall to reject) (Brainerd et al., 2003; Gallo, 2004). These results once again support the notion that signals within early visual regions support memory specificity and further show that the nature of this relationship is not fundamentally different in older adults (Koen et al., 2019). Rather, the overall strength of representations in midline occipital cortex seems to be reduced with age without changing the nature of the information contained within those representations.

In contrast, we found age differences in the relationship between target-thematic lure classification accuracy and behavioral memory discrimination in both lateral occipital and fusiform cortices. Young adults showed a positive relationship between neural discriminability and behavioral memory discriminability, whereas older adults showed a negative relationship. This age moderation effect emerged despite the smaller age difference in behavioral rejection rates for thematic lures compared with item lures. Age differences in this relationship suggest that the cognitive processes used for pattern classification differed across age groups, with a possible change in the type of information represented across age groups in these regions. The positive relationship in young adults likely reflects priming and recapitulation signals that are known to aid memory discrimination (Tootell et al., 1998). The negative relationship in older adults reflects processing associated with memory errors because older adults with more behavioral errors (i.e., poorer memory discrimination) showed better neural discrimination between old and new items. This result is consistent with studies showing that regions later in the visual hierarchy can be associated with false recognition (Garoff et al., 2005; Dennis et al., 2012; Gutchess and Schacter, 2012; Karanian and Slotnick, 2017). However, such activation is typically interpreted as reactivation of generic visual representations that lack sufficient detail to distinguish between old and new items, causing old and new information to be represented more similarly. The present result of better old/new discrimination in late visual regions in older adults with poorer memory is not completely consistent with this account, suggesting that memory errors can also be driven by processes that are different for targets and lures, not only those that make processing associated with these items more similar. Because this negative relationship 
Table 6. Whole-brain searchlight of target-item lure classification

\begin{tabular}{|c|c|c|c|c|c|c|}
\hline Region & $\mathrm{H}$ & $k$ & $t$ & $x$ & $y$ & $z$ \\
\hline \multicolumn{7}{|c|}{ Target-item lure classification: whole group $>$ theoretical chance } \\
\hline \multirow[t]{3}{*}{ Frontal pole } & M & 10 & 4.31 & -2 & 64 & 18 \\
\hline & $\mathrm{R}$ & 32 & 4.01 & 42 & 52 & 8 \\
\hline & $\mathrm{R}$ & 132 & 4.82 & 42 & 38 & 12 \\
\hline \multirow[t]{3}{*}{ Precentral gyrus } & $\mathrm{L}$ & 31 & 4.56 & -56 & 8 & 10 \\
\hline & $\mathrm{L}$ & 14 & 4.27 & -52 & -4 & 44 \\
\hline & $\mathrm{L}$ & 10 & 4.16 & -34 & -18 & 66 \\
\hline Postcentral gyrus & $\mathrm{L}$ & 25 & 4.68 & -40 & -30 & 58 \\
\hline Superior temporal gyrus & L & 46 & 4.65 & -50 & -42 & 4 \\
\hline Middle temporal gyrus & $\mathrm{L}$ & 33 & 4.82 & -62 & -48 & -4 \\
\hline Posterior cingulate & M & 12 & 4.25 & -4 & -48 & 32 \\
\hline \multirow[t]{4}{*}{ Precuneus } & L & 15 & 4.45 & -10 & -64 & 40 \\
\hline & M & 12 & 4.91 & -6 & -54 & 52 \\
\hline & M & 79 & 4.77 & 4 & -60 & 60 \\
\hline & $M$ & 10 & 4.75 & -4 & -62 & 12 \\
\hline Supramarginal gyrus & $\mathrm{L}$ & 147 & 5 & -46 & -34 & 46 \\
\hline \multirow[t]{2}{*}{ Lateral occipital cortex } & $\mathrm{L}$ & 14 & 4.15 & -44 & -72 & 20 \\
\hline & L & 12 & 3.94 & -26 & -76 & 30 \\
\hline Lingual gyrus & M & 13 & 4.31 & -4 & -68 & -2 \\
\hline Cuneus & M & 264 & 4.91 & 2 & -72 & 26 \\
\hline Calcarine cortex & M & 113 & 4.93 & 6 & -68 & 18 \\
\hline \multicolumn{7}{|c|}{ Target-item lure classification: young $>$ older adults } \\
\hline Inferior frontal gyrus & $\mathrm{L}$ & 19 & 3.69 & -44 & 4 & 18 \\
\hline \multirow[t]{3}{*}{ Postcentral gyrus } & $\mathrm{L}$ & 30 & 3.77 & -42 & -28 & 42 \\
\hline & $\mathrm{L}$ & 29 & 3.1 & -10 & -38 & 52 \\
\hline & $\mathrm{R}$ & 10 & 2.71 & -42 & -30 & 58 \\
\hline \multirow[t]{4}{*}{ Precuneus } & L & 16 & 2.84 & -18 & -68 & 38 \\
\hline & $M$ & 18 & 2.35 & 4 & -48 & 38 \\
\hline & M & 29 & 3.48 & 4 & -58 & 60 \\
\hline & $\mathrm{R}$ & 18 & 3.15 & 10 & -64 & 32 \\
\hline \multirow[t]{2}{*}{ Lingual gyrus } & $M$ & 1027 & 4.2 & 4 & -80 & -2 \\
\hline & $\mathrm{R}$ & 22 & 3.23 & 20 & -64 & -10 \\
\hline Calcarine cortex & $\mathrm{L}$ & 11 & 3.79 & -12 & -84 & 4 \\
\hline
\end{tabular}

Regions where target-item lure classification was above chance (upper portion) and those where classification accuracy was higher in young compared with older adults (lower portion). No region showed classification accuracy that was significantly better for older compared with young adults.

$H$, Hemisphere; $k$, cluster size in number of voxels; $t, t$-statistic of peak voxel, $x, y, z$, peak coordinates in MNI space.

emerged for target-thematic lure discrimination rather than target-item lure discrimination, it may represent a processing trade-off: older adults who represent old/new signals more strongly at a broad level to distinguish between targets and thematic lures do so at the expense of more subtle discriminations. These older adults then have stronger neural discriminability of targets and thematic lures, but poorer overall memory discrimination due to failure to reject item lures.

Outside of the ventral visual cortex, exploratory searchlight analyses showed broad discriminability of targets and thematic lures from signals across much of the brain, suggesting that converging signals from many regions contribute to these decisions that both age groups made with high accuracy. Although age deficits did not emerge for this neural discrimination in ROI analyses, portions of a number of cortical regions showed lower target-thematic lure classification accuracy in older compared with young adults. This finding is consistent with the small but significant age deficit in the behavioral accuracy of this discrimination. The set of regions where target and item lure signals were distinguishable was more limited and included midline occipital regions identified in the ROI analysis. Although univariate analyses have often shown both agerelated increases and decreases in activation (Dennis and Cabeza, 2008), classification for both item and thematic lures demonstrated age-related reductions and matched classification levels across age groups, but no regions where classifica- tion accuracy was higher in older compared with young adults. Therefore, using searchlight analyses, we find evidence of reduced neural discriminability in older compared with young adults across multiple levels of target-lure overlap.

By using pattern classification analyses during a memory retrieval task with multiple levels of target-lure overlap, we showed that regions of ventral visual cortex contain signals that differ between targets and thematic lures in both young and older adults. Additionally, midline occipital cortex also contained signals that differed between targets and item lures, consistent with the role of early visual regions in making fine-grained distinctions in memory. By linking classification accuracy to behavioral discrimination, we showed that, although weaker, the type of information represented by early visual signals is relatively consistent across age. Age moderation of the relationship between neural and behavioral discrimination in lateral occipital and fusiform cortices, however, suggests an age-related shift in the nature of the representations in these regions with age.

\section{References}

Balota DA, Cortese MJ, Duchek JM, Adams D, Roediger III HL, McDermott KB, Yerys BE (1999) Veridical and false memories in healthy older adults and in dementia of the Alzheimer's type. Cogn Neuropsychol 16: 361-384.

Bowman CR, Dennis NA (2016) The neural basis of recollection rejection: increases in hippocampal-prefrontal connectivity in the absence of a shared recall-to-reject and target recollection network. J Cogn Neurosci 28:1194-1209.

Bowman CR, Sine SL, Dennis NA (2017) Modulation of target recollection and recollection rejection networks due to retrieval facilitation and interference. Learn Mem 24:607-611.

Brainerd CJ, Reyna VF, Wright R, Mojardin AH (2003) Recollection rejection: false-memory editing in children and adults. Psychol Rev 110:762784.

Brodeur MB, Dionne-Dostie E, Montreuil T, Lepage M (2010) The bank of standardized stimuli (BOSS), a new set of 480 normative photos of objects to be used as visual stimuli in cognitive research. PLoS One 5:e10773.

Buchsbaum BR, Lemire-Rodger S, Fang C, Abdi H (2012) The neural basis of vivid memory is patterned on perception. J Cogn Neurosci 24:18671883.

Cabeza R, Daselaar SM, Dolcos F, Prince SE, Budde M, Nyberg L (2004) Task-independent and task-specific age effects on brain activity during working memory, visual attention and episodic retrieval. Cereb Cortex 14:364-375.

Carp J, Park J, Polk TA, Park DC (2011) Age differences in neural distinctiveness revealed by multi-voxel pattern analysis. Neuroimage 56:736743.

Dale AM (1999) Optimal experimental design for event-related fMRI.Hum Brain Mapp 8:109-114.

Davis SW, Dennis NA, Daselaar SM, Fleck MS, Cabeza R (2008) Qué PASA? The posterior-anterior shift in aging. Cereb Cortex 18:1201-1209.

Deese J (1959) On the prediction of occurrence of particular verbal intrusions in immediate recall. J Exp Psychol 58:17-22.

Dennis NA, Cabeza R (2008) Neuroimaging of healthy cognitive aging. In: The handbook of aging and cognition (Craik FEM, Salthouse TM, eds), pp 1-54. New York: Psychological Press.

Dennis NA, Bowman CR, Vandekar SN (2012) True and phantom recollection: an fMRI investigation of similar and distinct neural correlates and connectivity. Neuroimage 59:2982-2993.

Dennis NA, Bowman CR, Peterson KM (2014) Age-related differences in the neural correlates mediating false recollection. Neurobiol Aging 35: 395-407.

Desikan RS, Ségonne F, Fischl B, Quinn BT, Dickerson BC, Blacker D, Buckner RL, Dale AM, Maguire RP, Hyman BT, Albert MS, Killiany RJ (2006) An automated labeling system for subdividing the human cerebral cortex on MRI scans into gyral based ROIs. Neuroimage 31:968-980.

Folstein MF, Robins LN, Helzer JE (1983) The mini-mental state examination. Arch Gen Psychiatry 40:812.

Gallo DA (2004) Using recall to reduce false recognition: diagnostic and disqualifying monitoring. J Exp Psychol Learn Mem Cogn 30:120-128. 
Garoff RJ, Slotnick SD, Schacter DL (2005) The neural origins of specific and general memory: the role of the fusiform cortex. Neuropsychologia 43:847-859.

Gutchess AH, Schacter DL (2012) The neural correlates of gist-based true and false recognition. Neuroimage 59:3418-3426.

Gutchess AH, Welsh RC, Hedden T, Bangert A, Minear M, Liu LL, Park DC (2005) Aging and the neural correlates of successful picture encoding: frontal activations compensate for decreased medial-temporal activity. J Cogn Neurosci 17:84-96.

Henson RN, Rugg MD (2003) Neural response suppression, haemodynamic repetition effects, and behavioural priming. Neuropsychologia 41:263-270.

Johnson JD, Rugg MD (2007) Recollection and the reinstatement of encoding-related cortical activity. Cereb Cortex 17:2507-2515.

Karanian JM, Slotnick SD (2017) False memories for shape activate the lateral occipital complex. Learn Mem 24:552-556.

Kim H, Cabeza R (2007) Trusting our memories: dissociating the neural correlates of confidence in veridical versus illusory memories. J Neurosci 27:12190-12197.

Koen JD, Hauck N, Rugg MD (2019) The relationship between age, neural differentiation, and memory performance. J Neurosci 39:149-162.

Koutstaal W (2003) Older adults encode, but do not always use, perceptual details: intentional versus unintentional effects of detail on memory judgments. Psychol Sci 14:189-193.

Koutstaal W, Schacter DL (1997) Gist-based false recognition of pictures in older and younger adults. J Mem Lang 37:555-583.

Kuhl BA, Rissman J, Chun MM, Wagner AD (2011) Fidelity of neural reactivation reveals competition between memories. Proc Natl Acad Sci U S A 108:5903-5908.

Matzen LE, Taylor EG, Benjamin AS (2011) Contributions of familiarity and recollection rejection to recognition: evidence from the time course of false recognition for semantic and conjunction lures. Memory 19:1-16.

McCabe DP, Roediger HL 3rd, McDaniel MA, Balota DA (2009) Aging reduces veridical remembering but increases false remembering: neuropsychological test correlates of remember-know judgments. Neuropsychologia 47:2164-2173.

McCormick-Huhn JM, Bowman CR, Dennis NA (2018) Repeated study of items with and without repeated context: aging effects on memory discriminability. Memory 26:603-609.
Park DC, Polk TA, Park R, Minear M, Savage A, Smith MR (2004) Aging reduced neural specialization in ventral visual cortex. Proc Natl Acad Sci U S A 101:13091-13095.

Park J, Carp J, Kennedy KM, Rodrigue KM, Bischof GN, Huang CM, Rieck JR, Polk TA, Park DC (2012) Neural broadening or neural attenuation? investigating age-related dedifferentiation in the face network in a large lifespan sample. J Neurosci 32:2154-2158.

Polyn SM, Natu VS, Cohen JD, Norman KA (2005) Category-specific cortical activity precedes retrieval during memory search. Science 310:1963-1966.

Roediger HL, McDermott KB, Hintzman DL, Lindsay S, Rajaram S, Tulving E (1995) Creating false memories: remembering words not presented in lists. J Exp Psychol Learn Mem Cogn 21:803-814.

Schacter DL, Buckner RL (1998) Priming and the brain. Neuron 20:185-195.

Sheikh JI, Yesavage JA (1986) Geriatric depression scale (GDS) recent evidence and development of a shorter version. Clin Gerontol 5:165-173.

Slotnick SD, Schacter DL (2004) A sensory signature that distinguishes true from false memories. Nat Neurosci 7:664-672.

Tootell RB, Hadjikhani NK, Mendola JD, Marrett S, Dale AM (1998) From retinotopy to recognition: fMRI in human visual cortex. Trends Cogn Sci 2:174-183.

Watanabe T, Kimura HM, Hirose S, Wada H, Imai Y, Machida T, Shirouzu I, Miyashita Y, Konishi S (2012) Functional dissociation between anterior and posterior temporal cortical regions during retrieval of remote memory. J Neurosci 32:9659-9670.

Wechsler D (1997) WAIS-III WMS-III Technical Manual. San Antonio, TX: Psychological Corporation.

Wheeler ME, Petersen SE, Buckner RL (2000) Memory's echo: vivid remembering reactivates sensory-specific cortex. Proc Natl Acad Sci U S A 97:11125-11129.

Wing EA, Ritchey M, Cabeza R (2015) Reinstatement of individual past events revealed by the similarity of distributed activation patterns during encoding and retrieval. J Cogn Neurosci 27:679-691.

Winkler AM, Ridgway GR, Webster MA, Smith SM, Nichols TE (2014) Permutation inference for the general linear model. Neuroimage 92: 381-397. 